\title{
LA MÉTHODOLOGIE DE L'IMAGE PEUT-ELLE ÊTRE UTILE À LA RECHERCHE EN SCIENCES SOCIALES?
}

\section{Monique Haicault ${ }^{*}$}

Résumé: Si l'image n'a pas encore toute la place qui devrait lui revenir dans le champ des méthodologies en Sciences Sociales, c'est d'abord parce que son langage utilise un système de signes qui lui est spécifique, distinct de l'oral et de l'écrit. La méthodologie de l'image doit forger ses propres règles de collecte de données audiovisuelles, ses codes d'analyse et d'interprétation, afin de donner un sens sociologique à la "banalité " de ce qui est observé et enregistré. Elle doit aussi se plier aux conditions d'un média audiovisuel pour montrer des résultats de recherche dans un montage cohérent. L'article montre comment la réflexion s'alimente en particulier des apports de la Sémiologie et de ceux de l'Ecole de Palo Alto. Par sa nature épistémologique ce travail pousse la recherche sociologique à interroger ses catégories de collecte de données visuelles, à réajuster ses indicateurs, à préciser les objectifs de ce qu'elle veut transmettre. La méthodologie de l'image est donc utile à la production de connaissances en Sociologie. A partir d'exemples tirés des réalisations de l'auteure, l'article montre comment le corps, l'espace et le temps sont des catégories de la pratique sociale particulièrement adaptées aux approches audiovisuelles. Le corps au "Travail Domestique" ou en "Télétravail", l'espace dans "La gestion ordinaire de la vie en deux", "L'apprentissage du temps", au fondement de la prime socialisation.

Mots-clés: système de signes, Sémiologie, Palo Alto, catégories d'analyse, montage vidéo.

* Sociologue, LEST Aix en Provence.

Artigo recebido em 27 jun. 2003, aprovado em 30 ago. 2003. 
Dans la production de connaissances en Sciences Sociales, les images ont tardé à être reconnues comme des sources à part entière. C'est d'autant plus étonnant que nous sommes immergés depuis plusieurs décennies dans une société de l'image où celles-ci occupent dans la communication sociale un statut puissant. Mais le langage des images n'est pas une simple transposition de l'écrit dans le virtuel, pas plus que l'oral ne se transcrit directement en langage écrit. On se souvient des beaux textes de Barthes sur ce passage de l'oral au texte (1981). Un même travail est nécessaire à mener pour la saisie et la transmission de la connaissance du social par l'image.

L'usage nous informe que les images peuvent remplir plusieurs fonctions dans la démarche scientifique, ce qui les constitue comme une méthodologie à part entière. On en distingue au moins trois.

- elles peuvent servir de sources de données d'enquête. Elles sont déjà constituées, ou bien construites pour la recherche concernée. Dans les deux cas elles seront analysées comme n'importe quelle donnée qualitative de type verbal. Elles poseront au chercheur les mêmes problèmes et les mêmes exigences de rigueur dans le choix des catégories d'analyse. Il faudra de plus construire des catégories d'analyse des données visuelles, dont on dégagera quelques principes plus loin.

- elles peuvent faire avancer une recherche en s'emboîtant à d'autres techniques pour apporter leurs propres informations. Par exemple elles souligneront des liens entre dimensions, entre variables, liens qui peuvent avoir échappé aux instruments d'enquête classiques. Ainsi rendront-elles visible tout ce qui se rapporte au corps: les gestes, les postures, les enchaînements, les mises en scène, les objets et leur contexte; tout ce qui se rapporte aux temporalités: un processus de travail, la recomposition successive de tâches, leur durée, leur rythme, également les flux de présence/absence de figures sociologiques d'acteurs dans l'espace urbain, ou bien encore l'organisation spatio-temporelle d'un espace de travail, de vie domestique, de cité, de quartier. 
- enfin, les images peuvent raconter, montrer, transmettre un regard sur le monde social, faire voir la dynamique des rapports sociaux dans ce qui pourtant semble banal, naturel; montrer des acquis de socialisation, faire surgir ce qui fait sens pour le sociologue dans ce qui paraît relever de l'ordinaire et du quotidien. Celui-ci pourra tenter de transmettre des éléments de sa recherche sous forme de documentaire, ou sous la forme courte d'un article-vidéo. L'image prend alors pleinement sa fonction de communication pour devenir un outil de transmission sociale.

L'image a toujours été un moyen privilégié de communication sociale au cours de l'histoire de l'humanité. L'anthropologue Goody (1979) a rappelé qu'il n'existe pas de société sans images, ni même sans graphisme. Il a même affirmé et montré que les sociétés sans écriture, purement orales, possédaient déjà un savoir écrit, inscrit dans du graphisme, dans des signes (pictogrammes), autant de signesimages qui servaient à indiquer, mémoriser, exprimer, communiquer.

\section{Sociologie de l'image et sémiologie}

Le sociologue qui veut communiquer avec des images doit faire le choix de communiquer par des signes. Il doit s'aventurer à parler un autre langage que celui de l'écrit ou même de l'oral auxquels il est accoutumé à s'exprimer. La Science des Signes en tant que connaissance des liens entre signifiants et signifiés est relativement récente. Partant de la construction de ses «Eléments de sémiologie» Barthes (1964) a ouvert un registre de réflexions sur les liens entre la sociologie et l'image; ses mythologies en sont l'exemple le plus connu (1957).

Le rapport entre l'image, ses signes, ses significations et le social, est un rapport subtil et fluctuant car il s'inscrit dans une histoire et une culture sociales. Les signes semblent en constant changement par leurs signifiants - le monde tangible de la matière et des objets mais aussi par leurs signifiés - les contenus de signification. 
L'utilisation de signes pour transmettre une approche, un regard sur le social, se heurte donc à plusieurs difficultés. Notre expérience récente d'articles-vidéo sur «Le temps des villes» atteste que le décryptage sociologique des signes ne relève pas de l'évidence. Mal compris ne doivent-ils pas alors s'adosser à un bref support écrit plus explicite? Ce que tente de réaliser la forme article-vidéo que le montage ponctue de brefs intertitres explicatifs.

Le changement accéléré des sociétés industrialisées affecte la compréhension commune des images, leur signification, également leur forme et leur rythme. L'écriture filmique s'est accélérée, condensée - la publicité et les clips par exemple parlent un langage elliptique incompris de ceux qui n'ont pas suivi leur dynamique et qui s'est reversé en flux continu dans le langage filmique en général. De nouveaux codes transforment les métaphores, (le concret pour l'abstrait) - il paraitrait ridicule aujourd'hui de représenter le temps par une horloge, ou le travail par une sortie d'usine. Le recours aux métonymies (la partie pour le tout) doit s'adapter aux nouveaux «tout»s et aux nouvelles parties signifiantes de ces totalités. Un instrument de travail apte à signifier un poste de travail ou une qualification doit s'adapter aux transformations des technologies des systèmes productifs - même un ordinateur ne peut plus à lui seul signifier un poste de télétravail, comme il le faisait dans les années 80 (Haicault 1986). La culture de l'image envahit le travail sociologique et l'oblige à s'adapter.

Pourquoi les outils classiques des sciences sociales semblentils échapper à de telles remises en question concernant leur pertinence et leur actualité? A nos yeux un des apports scientifiques de l'image réside dans sa capacité de critique épistémologique des instruments et des catégories d'analyse classiques. Maintenue à l'écart de l'arsenal des méthodes légitimes, la méthodologie de l'image les protégerait en quelque sorte, de ce travail utile? Par leur inertie les indicateurs le mort saisissant le vif - ne filtrent-ils pas la nouveauté sociale et le changement, que l'image de son côté, ne peut éviter. 


\section{Sociologie de l'image et communication sociale}

Le développement de la méthodologie de l'image en Sociologie peut bénéficier des apports de l'Ecole de Palo Alto qui se combinent alors utilement à ceux de la sémiologie.

Les chercheurs de Palo Alto ont construit, durant la seconde moitié du $\mathrm{XX}^{\text {ème }}$ siècle aux Etats-Unis, une approche holistique et interactive de la communication qui s'est appuyée sur le corps, le corps comme langage (Batteson, 1981).

Le «Collège Invisible» affirme dans son postulat de base que la communication sociale humaine est inévitable et qu' elle utilise un grand nombre de canaux (son, parole, mots, ton, accent, gestes, corps paré, posture, position, regards, sourires, mimiques, tics). Il s'agit d'un "tout intégré", non fragmentable. Autour de Bateson, ces chercheurs, tous pluridisciplinaires, ont affirmé que le corps, la conscience, l'esprit, sont tous présents dans la communication humaine qui ne saurait se réduire à l'émission du seul langage articulé. Quoi de plus visuel par conséquent que ce corps qui exprime, qui se montre, qui dialogue, qui bouge et se tait, qui fabrique de l'événement, du temps et de l'espace (Haicault, 2000). De là à postuler que le social se manifeste toujours au travers de corps, de temps et d'espace et qu'il suffit de repérer, où, quand, comment, et quoi s'offre ainsi au regard, il n'y a qu'un pas à franchir pour le chercheur épris de compréhension et de transmission. Un regard certes qui fait retour sur ce qu'il capte et qui se nourrit d'informations adéquates. Bachelard (1934) n'affirmait-il pas «qu'il faut savoir pour voir». Ce qui nous conduit à renforcer l'idée que la méthodologie de l'image aussi bien pour recueillir des informations que pour transmettre un résultat de recherche en images, doit s'appuyer sur des connaissances susceptibles d'être transposables en images. C'est là une des difficultés et non des moindres, car elle suppose un transfert possible et compréhensible d'un langage dans un autre. Un vaste chantier s'est ainsi ouvert sur le travail de réflexion «corps, signes et communication», chantier qui attend que de nouveaux chercheurs s'y engagent. 
Si les apports des deux courants de réflexion - les signes et la communication interactive corporelle - ont pu étayer le bien fondé d'une démarche visant à saisir par l'image la corporéité de l'expérience sociale des acteurs, à la reconnaître pour mieux la montrer, d' autres courants de réflexion peuvent enrichir les domaines de connaissance de la sociologie de l'image. Les pistes ouvertes par ces écoles de pensée et par les travaux de Réseaux mis en place en sociologie au milieu des années 80 (Réseau National Pratiques audiovisuelles en Sciences Sociales 1986-1993), constituent un programme stimulant aussi bien pour la recherche que pour l'enseignement.

\section{La méthodologie de l'image à l'œuvre dans des articles vidéo}

On présentera pour finir quelques exemples d'application des principes proposés dans trois domaines: le corps, l'espace, le temps.

Le corps, conçu comme signifiant et objet de signification, est signe et langage. Il parle par ses postures, sa gestuelle, ses mimiques, son hexis corporelle, dans des interactions et dans des contextes porteurs d'un sens, qu'il faut parvenir à capter à l'enregistrement des images, puis au montage pour en souligner la pertinence sociologique. Le travail de montage - dont il ne sera toutefois pas question - joue un rôle décisif dans l'écriture du document vidéo, car il doit restituer des connaissances sociologiques avec des images «juste». Pas d'images bavardes dans un montage, pas de sensationnel non plus, mais du sens. Par exemple le document sur le travail domestique réalisé en 1976-1977 s'est attaché à saisir les corps de plusieurs figures de femmes, prises dans une activité de travail domestique, pour en briser la naturalité, la banalité coutumière, en «soulignant» les gestes et les instruments, la temporalité et le contexte de l'habitat. Au fil des enregistrements s'est fait jour l'idée qu'un même rapport social organisait le travail domestique de toutes ces femmes de la société française de ces années-là. Ceci nous avait conduite à élaborer théoriquement la notion même de rapport social de sexe, pour l'étendre plus tard à toutes les sphères d'activités. 
L'espace est aussi langage, il exprime des contenus culturels par ses agencements. Dans les années 80 un document intitulé «La vie en deux» traitait de l'articulation des différentes sphères d'activité de travail des femmes, au travers du portait de l'une d'entre elles qui signifiait quelque chose de commun à toutes les autres (Haicault, 1982). On a pu mettre en évidence non seulement «la conciliation acrobatique de ces virtuoses de la vie en deux», mais également la place tenue par l'espace, lui-même ensemble de signes, du simple fait qu'aucun espace n'est jamais socialement vide. Une grammaire de l'espace organisé par l'agencement des objets montre que l'espace de la maison est composé de traces matérielles significatives de l'appartenance sociale de la famille, ainsi que des objets de la consommation domestique de l'époque. Que ce soient les intérieurs familiaux, la chambre d'un enfant, le garage d'un télétravailleur à domicile, la cuisine d'une mécanicienne en confection, ou, plus largement, le quartier d'une ville ancienne, ces espaces peuvent se lire selon une grammaire de signes. Les signes de la temporalité urbaine et surtout de la politique de la ville se manifestent dans l'espace par des marqueurs qui, montrant les aménagements urbains par exemple, indiquent l'orientation de la politique urbaine (Haicault, 2002, 2003).

Le temps, si invisible qu'il soit, peut aussi se donner à lire dans des supports matériels qui sont autant de signifiants manifestés dans des indices visuels et sonores. Ainsi lors des enregistrements audiovisuels entrepris sur «L'apprentissage du temps» en milieu familial, une mère exprime la qualité "urgence" du temps par une simple métaphore gestuelle, le bras hachant l' air en rythme, une autre signifie à l'enfant le caractère implacable de l'heure par un mouvement de son corps tendu vers la porte, tandis qu' une autre mère inculque à son enfant la permission de goûter l'instant présent, sans même avoir à lui dire "prends ton temps"; l'absence de pression s'inscrivant dans une toute autre gestion de la totalité de la séquence temporelle du matin «Du réveil à la cloche» (Haicault, 1989).

La socialisation du temps peut encore se donner à voir dans la métonymie d'un objet, tel le cartable près de la porte d'entrée qui 
indique à l'enfant l'heure du départ pour l'école, ou par un indice, la lumière allumée brusquement dans la chambre ou l'odeur du pain grillé qui signifient «c'est l'heure de te lever». Autant de signes qui peuvent être lus comme des rituels temporels de passage.

Le temps s'inscrit donc socialement et visuellement dans des rituels. Pour les ouvrières en confection à domicile les rituels temporels de passage d'une activité à une autre, fabriquent du sens. Ils scandent les moments de la journée de travail dédoublée. Des rituels séparent le travail de la maison, du travail salarié effectué dans le même espace, ainsi la bouffée de cigarette avalée un bref instant - le corps immobile à l'image - avant la reprise auto-imposée du travail, fonctionne comme signe d'une liberté affirmée pour soi, pour soi seule, contre la pression temporelle de la double activité.

Comment montrer à l'image des pratiques qui paraissent tellement banales, dès lors qu'elles sont enfouies dans l'ordinaire des inculcations culturelles? La méthodologie de l'image entraîne à sortir de l'ethnocentrisme, à forger un regard depuis l'extérieur.

La sociologie audiovisuelle produit des documents visuels et sonores appuyés sur une recherche qui forcément la précède. Elle ne raconte pas des événements comme le fait le cinéma du réel, le direct ou un reportage. Elle n'illustre pas non plus un discours comme on le voit dans les premiers documentaires sociologiques de l'après-guerre qui comportaient un commentaire très didactique, nécessaire à un moment de l'histoire de l'écriture filmique et surtout de l'éducation du public. La sociologie audiovisuelle actuelle ne démontre pas, elle prend le risque de montrer. Elle cherche à transmettre un autre regard sur le monde quotidien. Dans un corps à corps avec les images, les sons, les signes, en recherche aussi vers un langage en phase avec la culture de l'image, elle cherche à briser les présupposés sociaux, moraux, religieux, disciplinaires.

Si qualitative qu'elle paraisse la méthodologie de l'image n'échappe toutefois pas aux exigences de vigilance de la démarche, telles qu'elles ont été inventoriées par Bachelard et plus tard par les auteurs du métier de sociologue (Bourdieu, Chamboredon, Passeron, 
1968). Ces exigences sont applicables à toutes les méthodes des Sciences Sociales, les nouvelles comme les plus anciennes. Pour l'image «les règles de la méthode» sont encore à développer. Elles sont aussi à enseigner, car elles ont leur place au sein de l'arsenal des instruments de collecte et de diffusion de la science sociale. Les chercheurs et les étudiants qui la pratiquent, celles et ceux qui l'enseignent, sont convaincus que cette nouvelle méthodologie stimule la réflexion sociologique et contribue à la faire progresser.

La sociologie audiovisuelle possède donc la capacité d'une authentique méthode d'observation. Filmer, c'est reconnaître l'autre dans son expérience quotidienne, anonyme, oubliée, sans mémoire, ensevelie sous le flot sans fin de l'événement qui tient toujours la première place. Sachant toutefois qu'on ne voit, qu'on ne reconnaît que des facettes, des parties, des formes, du fait de la nécessité épistémologique du découpage de l'objet d'étude. Sans oublier jamais que montrer c'est aussi cacher, dire c'est également taire et faire taire, écouter c'est aussi rester en partie sourd. Mais la méthodologie de l'image ne détient - elle pas une qualité plus rare encore, celle de pouvoir communiquer par des signes et dans des rapports fragiles certes, entre images, parole et écriture, un peu plus de conscience du monde que nous contribuons à créer.

Resumo: Se a imagem ainda não tem seu devido lugar no campo da metodologia das Ciências Sociais, deve-se ao fato de que sua linguagem utiliza um sistema de signos que lhe é próprio, distinto da oralidade e da escritura. A metodologia da imagem deve criar suas próprias regras de coleta de dados audiovisuais, seus códigos de análise e de interpretação, a fim de dar sentido sociológico à "banalidade" daquilo que é observado e registrado. Ela deve também se render às condições de uma mídia audiovisual para mostrar seus resultados e pesquisa em uma montagem coerente. $\mathrm{O}$ artigo mostra como a reflexão se alimenta, em particular, dos aportes da Semiologia e daqueles da Escola de Palo Alto. Por sua natureza epistemológica este trabalho conduz a pesquisa sociológica a interrogar suas categorias de coleta de dados visuais, a reajustar seus indicadores e a precisar os objetivos daquilo que ela pretende transmitir. A metodologia da imagem é, portanto, útil à produção de conhecimentos em Sociologia. 
Palavras-chave: sistemas de signos, Semiologia, Palo Alto, categorias de análise, vídeo-montagem.

Abstract: Video has not already found a good place in the field of methodology of Social Sciences. His language is different both from the oral and of writing form of expression, because it is a specific system of signs. This technique must find its own data rules, its own categories of analysis and meaning. Its must break the evidence of reality. If the sociologist wants to show his research, he must accept the rules of media and produce an adequate video. For that, the knowledge of Semiology and of Palo Alto College is really important to know. For that, the audio-visual methodology is surely useful to bust forward the sociological practice. From his own shots and videos the author indicates how tree categories of social experience are used by audio-visual methodology: the body in "The domestic work", the space in "The ordinary management of life cut in two", and the learning of social time in family life, during the prime socialisation.

Key-words: system of signs, Semiology, Palo Alto, categories of analysis, video.

\section{Bibliographie}

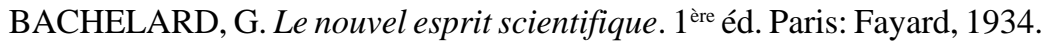

BATTESON, G. et al. La nouvelle communication. Paris: Ed. du Seuil, 1981. (Collection Points).

BARTHES, R. Mythologies. Paris: Ed. du Seuil, 1957.

Eléments de sémiologie. Communication, n. 4, 1964. . Le grain de la voix. Paris: Ed du Seuil, 1981.

BOURDIEU, P.; CHAMBOREDON, J. C.; PASSERON, J. C. Le métier de sociologue. Paris: Ed de Minuit, 1968.

GOODY, J. La raison graphique: la domestication de la pensée sauvage. Paris: Ed de Minuit, 1979.

HAICAULT, M. Marie Thérèse, la vie en deux. 1982. Vidéo 50 min.

. Vivre et travailler chez soi. 1986. Vidéo N/B de 21 min. sur le travail à domicile et le télétravail. 
HAICAULT, M. Famille, école et temps, du réveil à la cloche: des modes familiaux de socialisation. Revue Suisse de Sociologie, n. spécial, 1999.

. L'expérience sociale du quotidien, corps, espace, temps. Ottawa: Presses de L'Université d'Ottawa, 2000.

. Vivre en ville: Rennes en Bretagne. 2002. Vidéo 14 min.

. Marseille: des femmes dans les lieux et les temps quotidiens. 2003. Vidéo 21 min.

RESEAU NATIONAL PRATIQUES AUDIOVISUELLES EN SCIENCES DE LA SOCIETÉ. Cahiers, Nantes, n. 1, 1987.

. Cahiers, La parole dans le film, Aix en Provence, n. 2, 1988

. Cahiers, La caméra sur le terrain, Vaucresson, n. 3, 1989. 
